\title{
Functional Analysis of Conformational Changes of Phosphofructokinase
}

\author{
Teresa Ruiz
}

Department of Molecular Physiology and Biophysics, University of Vermont, Burlington, USA

The glycolytic flux is tightly regulated in unicellular and multicellular organisms under anaerobic conditions. A main regulatory step is centered around the behavior of phosphofructokinase (PFK), which catalyzes the phosphorylation of fructose-6-phosphate (F6P) to fructose-1,6-bisphosphate in the presence of adenosine triphosphate (ATP). PFK shows a species dependent subunit composition, oligomerization state, and specific kinetic behavior. Monomer sizes range from $34 \mathrm{kDa}$ in prokaryotes to $110 \mathrm{kDa}$ in eukaryotes. The diversity of composition and oligomerization state allows for subtle differences in the catalytic and regulatory mechanism of this enzyme in different organisms. The most knowledge has been obtained for the bacterial PFKs, which are homotetramers with a molecular mass of $140 \mathrm{kDa}$. A combination of X-ray crystallographic and biochemical data has provided an understanding of the conformational changes that occur between the active and inactive states and the role of the only two bacterial effectors [1]. Inactivation is achieved by a $7^{\circ}$ rotation of the subunits in the plane perpendicular to the catalytic surface. Eukaryotic PFKs have a higher level of regulatory complexity facilitated by an evolutionary process of gene duplication and fusion of the bacterial gene that has given rise to a "double-size" eukaryotic enzyme.

Among all the eukaryotic PFKs, the one from $S$. cerevisiae (ScPFK) is the best characterized. This enzyme is a hetero-octamer of $835 \mathrm{kDa}$, composed of $4 \alpha$ - and $4 \beta$-subunits of approximately $105 \mathrm{kDa}$ each. Like its prokaryotic counterpart, it shows cooperativity for F6P and non-cooperativity for ATP. Most insights into the structural/functional mechanism of these enzymes have been obtained by 3DEM. The $4 \alpha$-subunits of the $S c$ PFK form the inner core of the enzyme onto which the $4 \beta$-subunits are bound [2]. These 3DEM studies permitted to correctly infer the composition and the localization of the catalytic binding region [3] and combined with molecular replacement aided the determination the X-ray structure of the ScPFK truncated tetramer in the presence of F6P [4]. The cryo 3DEM structure of $S c$ PFK in the inactive state [5], in combination with studies of other yeast structures in different states, has manifested a strong correlation between structural conformation and kinetic behavior. The structural studies have shown that minor subunit rearrangements influence the rotation between the tetramers in the octameric complex, correlated with the kinetic behavior of the enzyme. However, there is still a lack of understanding of the small structural changes that lead to this large conformational change.

We have analyzed the ScPFK in the presence of either F6P or ATP to understand the structural changes taking place when the enzyme switches from the active to the inactive state. Cryo 3DEM data were collected for the enzyme in both states (Fig. 1, Top) and 3D reconstructions, with $1.3 \mathrm{~nm}$ resolution, were calculated by 3D reference based projection alignment methods using Radon transform algorithms (Fig. 1, Center). Visualizations were carried out using Chimera [6] and segmentations were computed using the Segger watershed algorithm [7] distributed with Chimera. An initial segmentation with 5 rounds of smoothing divided the $S c$ PFK in the active state into 16 small subdomains that correlated well, within the homology regions, with those of its $E$. coli counterpart. These could be merged to produce 8 well-defined subunits (Fig. 1, Bottom). These results permitted the visualization and characterization of the catalytic interface (interface between the $\mathrm{N}$-terminal regions of the $\alpha$ - and $\beta$-subunits) and the octamerization interface (interface between the $\mathrm{C}$-terminal regions of $\alpha$-subunits of two tetramers). Two 
further rounds of smoothing merged groups of 4 subunits showing the octameric enzyme divided into two distinct tetramers (top/bottom). A similar procedure applied to the ScPFK in the inactive state showed that the interactions between the $\alpha-\alpha$ subunits at the octamerization interface are much stronger in the inactive state (Fig. 1, arrows). In addition, after several rounds of smoothing, the structure failed to segment into two distinct tetramers (top/bottom). This technique is allowing us to dissect the small conformational changes occurring during ScPFK activation and will aid our efforts to model the 3D structure of the whole octameric enzyme, using X-ray data and structural prediction methods.

\section{References:}

[1] P.R. Evans, in Proceedings of the R.A. Welch Found. Conf. on Chem. Research 36. (1992) p39.

[2] T. Ruiz, et al. J Struct Biol, 136 (2001) p167.

[3] T. Ruiz, et al. J Struct Biol, 143 (2003) p124.

[4] K Banaszak, et al. J Mol. Biol, 407 (2011) p284.

[5] M Barcena, et al. J Struct Biol, 159 (2007) p135.

[6] E.F. Pettersen, et al. J Comput Chem., 13 (2004) p1605.

[7] G. Pintilie, , et al. J Struct Biol, 170 (2010) p427.

[8] This work was supported by Grants R01 GM069551and R01 GM069551-S1 from the National Institute of Health.

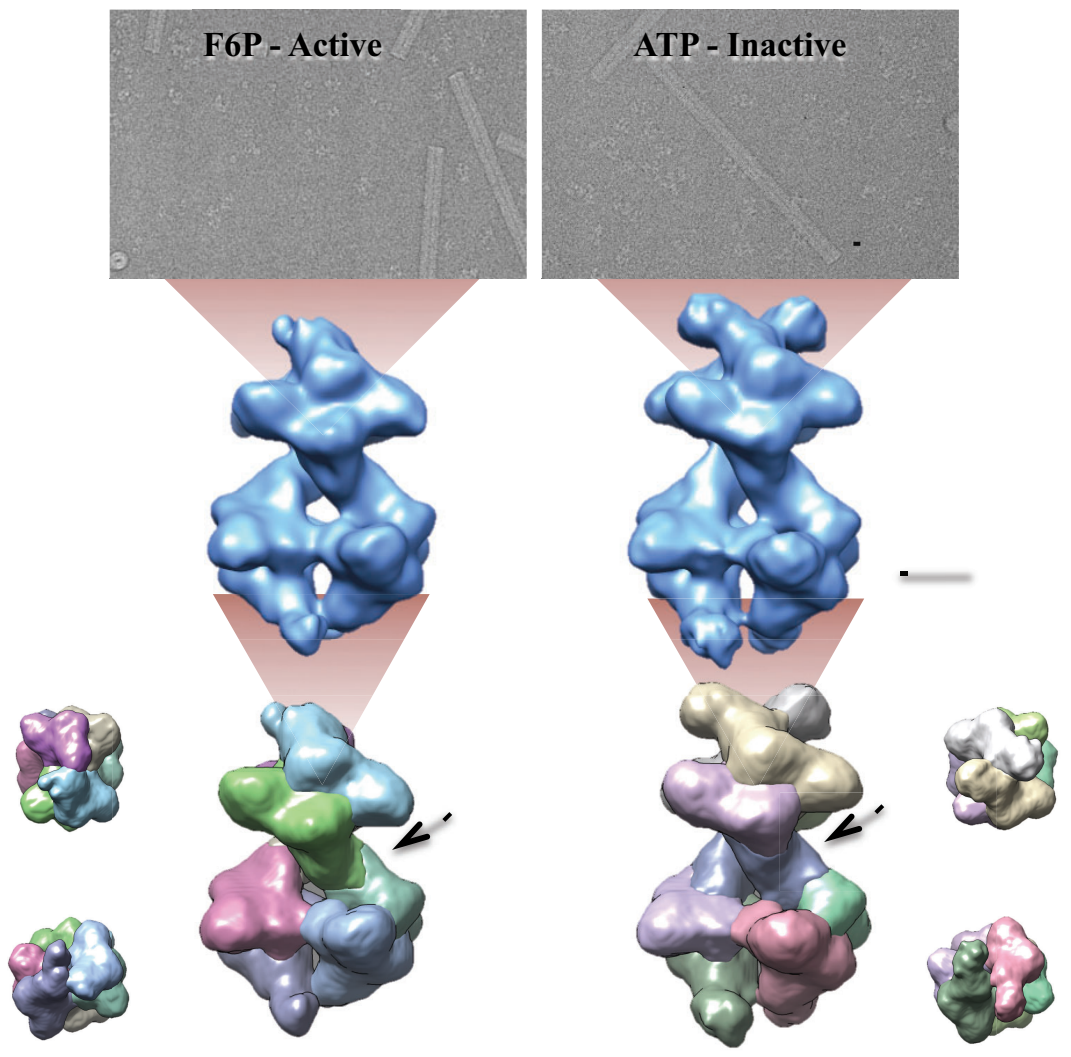

Figure 1. (Top) Cryo-electron micrographs of $S c P F K$, Bar $=100 \mathrm{~nm}$; (Center) 3DEM reconstructions, Bar=5nm; (Bottom) Segmentation of the 3D structures front, top and bottom views are shown. Each of the 8 subunits is shown in a different color. Arrows point to the $\alpha-\alpha$ subunit interface at the core of the enzyme. 